\title{
Optimal Power Allocation and Scheduling for Two-Cell Capacity Maximization
}

\author{
Anders Gjendemsjø $\varnothing^{*}$, David Gesbert ${ }^{\dagger}$, Geir E. Øien*, and Saad G. Kiani ${ }^{\dagger}$ \\ *Dept. of Electronics and Telecom., Norwegian Univ. of Science and Technology, \\ 7491 Trondheim, Norway, Email: \{gjendems, oien\}@iet.ntnu.no \\ ${ }^{\dagger}$ Mobile Communications Department, Institute Eurécom, \\ 06560 Sophia-Antipolis, France, Email: \{gesbert, kiani\}@eurecom.fr
}

\begin{abstract}
We consider the problem of optimally allocating the base station transmit power in two neighboring cells for a TDMA wireless cellular system, to maximize the total system throughput under interference and noise impairments. Employing dynamic reuse of spectral resources, we impose a peak power constraint at each base station and allow for coordination between the base stations. By an analytical derivation we find that the optimal power allocation then has a remarkably simple nature: Depending on the noise and channel gains, transmit at full power only at base station 1 or base station 2 , or both.

Utilizing the optimal power allocation we study optimal link adaptation, and compare to adaptive transmission without power control. Results show that allowing for power control significantly increases the overall capacity for an average user pair, in addition to considerable power savings. Furthermore, we investigate power adaptation in combination with scheduling of users in a time slotted system. Specifically, the capacity-optimal singlecell scheduler [1] is generalized to the two-cell case. Thus, both power allocation and multiuser diversity are exploited to give substantial network capacity gains.
\end{abstract}

\section{INTRODUCTION}

The need for ever higher spectrum efficiency motivates the search for system-wide optimization of the wireless resources. A key example of multicell resource allocation is that of power control which serves as means for both battery savings at the mobile, and interference management. Traditional power control solutions are designed for voice-centric networks, hence aiming at guaranteeing a target signal to noise and interference ratio (SNIR) level to the users [2]-[4]. In modern wireless data networks, adaptive coding and modulation with power control [5], [6] is implemented, and throughput maximization becomes a more relevant metric.

The simultaneous optimization of transmission rates and power with the aim of maximizing the multicell sum capacity is a difficult problem [7]-[10]. Considering the problem of optimally allocating the transmit power for $N$ active communication links, an iterative algorithm to maximize the network capacity measured in information bits/s/Hz is given in [7]. Unfortunately, this algorithm can converge to a highly suboptimal solution, thus an approximation to the network capacity is proposed and a fixed-point iteration solution is derived. However, this approximation prohibits turning off any base station, even if this is optimal due to interference.

Under the assumption of an interference limited system, i.e., neglecting noise sources, iterative algorithms are proposed to maximize the network capacity for the case of individual link power constraints [8] and a sum power constraint [9]. In [10] it is assumed that each base station, when it transmits, transmits with maximum power $P_{\max }$. Which base stations that should be active at each time slot is decided according to a rate maximization objective. However, no proof of optimality is given for the on/off power allocation.

In [11] transmit power allocation for a downlink two-user interference channel is studied under a sum transmit power constraint and the assumption of symmetric interference. The derived power allocation depends on the level of interference; when the inference is above a certain threshold the total power is allocated to the best user. For interference less than the threshold, the available power is divided among the two users according to a water-filling principle. However, due to the sum power constraint and symmetry of interference assumption these results are not readily applicable for two-cell power allocation, where it is more reasonable to assume individual power constraints and that the received interference will be different for different users.

In this paper we tackle the problem of analytically finding a closed form solution for power allocation in a simple two-cell network, without resorting to the restricting assumptions of interference limiting or symmetry. The optimal power allocation is shown to be remarkably simple; transmit at full power only at base station 1 , or only at base station 2 , or both. Using the derived power allocation scheme, link adaptation with power control is compared to adaptive transmission without power control, showing that substantial sum throughput gains can be achieved by introducing power adaptation for an average user pair. Further, we combine the derived power allocation with scheduling of users [1], [12], considering both Round Robin and generalizations of the single-cell maximum capacity scheduler [1] to the two-cell case, thus exploiting both power control and multiuser diversity to achieve increased capacity.

The remainder of our paper is organized as follows. We introduce the wireless system model under investigation in Section II. In Section III we derive the optimal power allocation for two-cell capacity maximization. Section IV combines the derived power allocation scheme with scheduling of users in order to maximize the system capacity. Numerical results and plots are presented in Section V. Finally, conclusions and discussions are given in Section VI. 


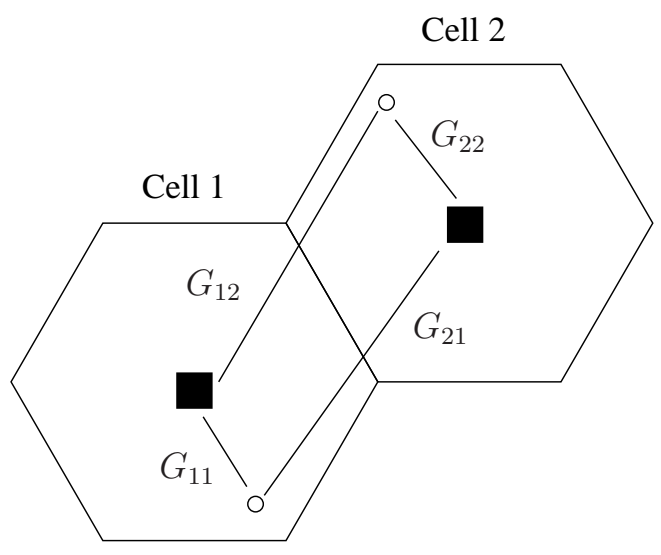

Fig. 1. Two-cell wireless system model. Base stations are shown as solid squares and users as circles. To each link the associated channel gain $G_{n i}$ is shown.

\section{SySTEM MODEL}

We consider a two-cell system in which two neighboring base stations communicate with mobile terminals over a coverage area as depicted in Fig. 1. Each of the two base stations transmit different data to two different receiving mobile users at the same time using the same spectral resource, leading in general to an interference and noise impaired system. The communication links may be a combination of up- and downlinks. Although an hexagonal cell layout is shown in Fig. 1, we emphasize that our analysis is valid for any geometry, even for non-cellular systems such as ad-hoc networks. For the sake of presentation, and without loss of generality [13], we focus on downlink transmission. The data destined for user $u_{n}$ is transmitted with power $P_{n}, n=1,2$. Each base station is assumed to operate under a peak power constraint,

$$
0 \leq P_{n} \leq P_{\max }, \quad n=1,2 .
$$

Denote by $G_{n i}(t)$ the channel power gain to the selected mobile user $u_{n}(t)$ in cell $n$ from the base station in cell $i$ at time $t$. We will suppress the time index from now on, concentrating on one arbitrary time slot. The channel gains are assumed to be constant over each such time slot. Note that the gains $G_{n n}$ correspond to the desired communication links, whereas the $G_{n, i, n \neq i}$ correspond to the unwanted interference links. Assuming the transmitted symbols to be independent random variables with zero mean and unit variance, the signal to noise-plus-interference ratio (SNIR) for each user is given by,

$$
\mathrm{SNIR}_{u_{1}}=\frac{P_{1} G_{11}}{\sigma_{1}^{2}+P_{2} G_{12}}, \mathrm{SNIR}_{u_{2}}=\frac{P_{2} G_{22}}{\sigma_{2}^{2}+P_{1} G_{21}},
$$

where $\sigma_{1}^{2}$ and $\sigma_{2}^{2}$ are the variances of the independent zeromean additive white gaussian noise (AWGN) in cell 1 and 2, respectively.

Under the assumption that capacity-achieving codes for AWGN channels are employed, the achievable rate (in information bits $/ \mathrm{s} / \mathrm{Hz}$ ) of user $u_{n}$ is given by

$$
R_{u_{n}}=\log _{2}\left(1+\mathrm{SNIR}_{u_{n}}\right) .
$$

From (2) and (3) the total achievable throughput $R=R_{u_{1}}+$ $R_{u_{2}}$ is given by

$$
R=\log _{2}\left(1+\frac{P_{1} G_{11}}{\sigma_{1}^{2}+P_{2} G_{12}}\right)+\log _{2}\left(1+\frac{P_{2} G_{22}}{\sigma_{2}^{2}+P_{1} G_{21}}\right) .
$$

Finally, we note that our system model with (possibly different) noise levels $\sigma_{1}^{2}$ and $\sigma_{2}^{2}$ also facilitates the modeling of other interfering gaussian sources disturbing the users differently, contrary to [10], [11].

\section{Transmit Power Analysis}

This section presents the general optimal power allocation scheme $\left(P_{1}^{*}, P_{2}^{*}\right)$ which has as inputs the channel gains $\left\{G_{n i}\right\}$, and the AWGN variances $\sigma_{1}^{2}$ and $\sigma_{2}^{2}$. We search for the optimal power allocation by approaching the following optimization problem,

$$
\left(P_{1}^{*}, P_{2}^{*}\right)=\arg \max _{\left(P_{1}, P_{2}\right) \in \Omega} R,
$$

where $\Omega=\left\{P_{1}, P_{2} \mid 0 \leq P_{1}, P_{2} \leq P_{\max }\right\}$ is the feasible set and $R$ is given in (4). Since $\Omega$ is a closed and bounded set and $R: \Omega \rightarrow \mathbb{R}$ is continuous, (5) has a solution [14, Theorem $0.3]$.

\section{A. Trivial solutions}

By inspection of (4) we can identify some trivial (not necessarily unique) solutions of (5). First, if the system is noise limited, i.e., the interference can be neglected, then $\left(P_{1}^{*}, P_{2}^{*}\right)=\left(P_{\max }, P_{\max }\right)$. Second, if the communication link of user 1 is blocked, i.e., $G_{11}=0$, then $\left(P_{1}^{*}, P_{2}^{*}\right)=\left(0, P_{\max }\right)$; vice versa for user 2 . Finally, for the case of an interference limited system, we see that $R \rightarrow \infty$ if only one of the two base stations is turned on. However, in our analysis we will assume that some noise is present, as in all practical systems.

\section{B. General solutions}

In this subsection we assume $G_{n i}>0, \forall n, i$. Then in order to find $\left(P_{1}^{*}, P_{2}^{*}\right)$ we first prove the following lemma.

Lemma 1: The optimal transmit power allocation will have $P_{1}$ or $P_{2}$ equal to $P_{\max }$.

Proof: From (4) we have that, for $\alpha>1$ and $\left(P_{1}, P_{2}\right) \in$ $\Omega$ :

$$
\begin{aligned}
& R\left(\alpha P_{1}, \alpha P_{2}\right)= \\
& \log _{2}\left(\left(1+\frac{P_{1} G_{11}}{\frac{\sigma_{1}^{2}}{\alpha}+P_{2} G_{12}}\right)\left(1+\frac{P_{2} G_{22}}{\frac{\sigma_{2}^{2}}{\alpha}+P_{1} G_{21}}\right)\right) \\
& >R\left(P_{1}, P_{2}\right) .
\end{aligned}
$$

Hence, the solution of (5) will have $P_{1}$ or $P_{2}$ equal to $P_{\max }$.

By Lemma 1, the optimal power allocation is found among the following alternatives:

- Extreme points on the boundaries of $\Omega: P_{2}=P_{\max }$ or $P_{1}=P_{\max }$, i.e., $P_{1}$ 's or $P_{2}$ 's corresponding to $\frac{\partial R\left(P_{1}, P_{\max }\right)}{\partial P_{1}}=0$ or $\frac{\partial R\left(P_{\max }, P_{2}\right)}{\partial P_{2}}=0$, respectively.

- Corner points of $\Omega:\left(P_{\max }, 0\right)$, or $\left(0, P_{\max }\right)$, or $\left(P_{\max }, P_{\max }\right)$. 
Since the logarithm is a monotonically increasing function we look for extreme points on the boundary by considering

$$
Q\left(P_{1}, P_{2}\right)=\left(1+\frac{P_{1} G_{11}}{\sigma_{1}^{2}+P_{2} G_{12}}\right)\left(1+\frac{P_{2} G_{22}}{\sigma_{2}^{2}+P_{1} G_{21}}\right) .
$$

Now, by differentiating $Q\left(P_{1}, P_{\max }\right)$ with respect to $P_{1}$ we find

$$
\frac{\partial Q}{\partial P_{1}}=\frac{C P_{1}^{2}+2 D P_{1}+E}{F},
$$

where

$$
\begin{aligned}
C & =G_{11} G_{21}^{2}, \\
D & =G_{11} G_{21} \sigma_{2}^{2}, \\
E & =-P_{\max } G_{21} G_{22}\left(\sigma_{1}^{2}+P_{\max } G_{12}\right) \\
& +G_{11} \sigma_{2}^{2}\left(\sigma_{2}^{2}+P_{\max } G_{22}\right), \\
F & =\left(\sigma_{1}^{2}+P_{\max } G_{12}\right)\left(\sigma_{2}^{2}+P_{1} G_{21}\right)^{2} .
\end{aligned}
$$

From (9d) $F$ is seen to always be positive, hence $P_{1}$ such that $\frac{\partial Q}{\partial P_{1}}=0$ can be found as the solution to $C P_{1}^{2}+2 D P_{1}+E=0$, yielding

$$
P_{1}=\frac{1}{C}\left(-D \pm \sqrt{D^{2}-C E}\right) .
$$

From (10) we are only interested in real-valued $P_{1} \in\left[0, P_{\max }\right]$. By inspecting (10) we realize that a real and non-negative $P_{1}$ can only occur for $E \leq 0$, since $C, D>0$. Denote such a $P_{1}$ by $P_{1}^{\mathrm{rp}}$. To see whether $P_{1}^{\mathrm{rp}}$ corresponds to a maximum or minimum for $Q\left(P_{1}, P_{\max }\right)$ we calculate the second derivative $\frac{\partial^{2} Q}{\partial P_{1}^{2}}$

$$
\frac{\partial^{2} Q}{\partial P_{1}^{2}}=\frac{2 P_{\max } G_{22} G_{21}\left(G_{21} \sigma_{1}^{2}+G_{21} P_{\max } G_{12}-G_{11} \sigma_{2}^{2}\right)}{\left(\sigma_{1}^{2}+P_{\max } G_{12}\right)\left(\sigma_{2}^{2}+P_{1} G_{21}\right)^{3}} .
$$

$\frac{\partial^{2} Q}{\partial P^{2}}$ is seen to be non-negative if the following inequality is satisfied:

$$
G_{21}\left(\sigma_{1}^{2}+P_{\max } G_{12}\right) \geq G_{11} \sigma_{2}^{2} .
$$

Now, by inspecting (9c) we see that $E \leq 0$ implies that

$$
P_{\max } G_{22} G_{21}\left(\sigma_{1}^{2}+P_{\max } G_{12}\right) \geq G_{11} \sigma_{2}^{2}\left(\sigma_{2}^{2}+P_{\max } G_{22}\right) .
$$

Dividing by $P_{\max } G_{22}$ on both sides of (13) we obtain

$$
\begin{aligned}
G_{21}\left(\sigma_{1}^{2}+P_{\max } G_{12}\right) & \geq G_{11} \sigma_{2}^{2}\left(1+\frac{\sigma_{2}^{2}}{P_{\max } G_{22}}\right) \\
& \geq G_{11} \sigma_{2}^{2},
\end{aligned}
$$

thus showing $\frac{\partial^{2} Q}{\partial P_{1}^{2}} \geq 0 . Q\left(P_{1}, P_{\max }\right)$ is then convex with respect to $0 \leq P_{1} \leq P_{\max }$ and $P_{1}^{\mathrm{rp}}$ is a minimum point for $Q\left(P_{1}, P_{\max }\right)$. Due to symmetry, the above analysis also hold for $P_{2}$, and we conclude that $\left(P_{1}^{*}, P_{2}^{*}\right)$ is found in the set of corner points: $\Delta \Omega=\left\{\left(P_{\max }, 0\right),\left(0, P_{\max }\right),\left(P_{\max }, P_{\max }\right)\right\}$. The optimal power allocation (also including the trivial solutions from Section III-A) can then be stated as

$$
\left(P_{1}^{*}, P_{2}^{*}\right)=\arg \max _{\left(P_{1}, P_{2}\right) \in \Delta \Omega} R\left(P_{1}, P_{2}\right) .
$$

By inspection of (15) we see that of the two users in question, the user with the highest signal to noise ratio (SNR), defined as $\frac{G_{n n}}{\sigma^{2}}$, will always receive transmission at full power $P_{\max }$. For $\left(P_{1}^{n}, P_{2}\right)=\left(P_{\max }, P_{\max }\right)$ this is trivially true. Further, from (4), the choice of $\left(P_{1}, P_{2}\right)=\left(P_{\max }, 0\right)$ or $\left(0, P_{\max }\right)$ is decided by each user's SNR alone, since there will be no interference for these power allocations.

Since one of the base stations may be turned off the spectrum reuse will be dynamic. From a system perspective, the power allocation in (15) is intended to be used for two given users in a given time slot. As such, it is natural to combine this power allocation scheme with a scheduling algorithm which we examine in the next section.

\section{SCHEDULING}

In this section the optimal power allocation is combined with scheduling of users in a time-slotted system. The proposed power allocation can be used with any network scheduling algorithm. As examples, we consider Round Robin, maximum SNR scheduling, and the novel two-cell maximum capacity scheduling.

\section{A. Round Robin Scheduling}

Round Robin scheduling [15] is the simplest scheduling algorithm for users in a wireless system. Users are given time slots on a turn by turn basis, ensuring resource fair scheduling. In our two-cell scenario we schedule, at each time slot, one user from each cell using full power. This will be considered as the baseline case. To increase the system capacity we introduce power control at each time slot by solving (5) for each pair of users supplied by the Round Robin scheduling.

\section{B. Maximum SNR Scheduling}

By exploiting the inherent channel variations in wireless systems, maximum SNR scheduling seeks to maximize the cell throughput by transmitting to the mobile user with the best channel in every time slot [1], [16]. Let $U_{1}$ and $U_{2}$ denote the number of users in cell 1 and cell 2, respectively. Then, as above, by scheduling one user from each cell, users $u_{1}^{*}$ and $u_{2}^{*}$ are selected if

$$
\begin{aligned}
& u_{1}^{*}=\arg \max _{1 \leq u_{1} \leq U_{1}} \frac{G_{11}}{\sigma_{1}^{2}}, \\
& u_{2}^{*}=\arg \max _{1 \leq u_{2} \leq U_{2}} \frac{G_{22}}{\sigma_{2}^{2}} .
\end{aligned}
$$

As above, we can increase the system capacity by applying the power control (15) to users $u_{1}^{*}$ and $u_{2}^{*}$.

\section{Two-Cell Maximum Capacity Scheduling}

By taking interference and power control into account, we generalize the single-cell maximum SNR scheduler [1] to the two-cell case. The extension is a two-step process, jointly optimizing the user selection and power levels to maximize the network capacity. The maximum capacity scheduling algorithm can then be stated as follows. At each time slot $s$,

1) For each pair of users $\left(u_{1}, u_{2}\right)$ : Find the optimal power allocation $\left(P_{1}^{*}, P_{2}^{*}\right)$ from (15), and then store corresponding achievable throughput $R\left(u_{1}, u_{2}\right)$. 
2) Schedule the users $\left(u_{1}^{*}, u_{2}^{*}\right)$ that have maximum sum throughput $R\left(u_{1}, u_{2}\right)$ and transmit with power levels $\left(P_{1}^{*}, P_{2}^{*}\right)$.

Mathematically the above algorithm can be formulated as

$$
\left(u_{1}^{*}, u_{2}^{*}, P_{1}^{*}, P_{2}^{*}\right)=\arg \max _{\substack{1 \leq u_{1} \leq U_{1} \\ 1 \leq u_{2} \leq U_{2} \\\left(P_{1}, P_{2}\right) \in \Delta \Omega}} R .
$$

Although the exhaustive search in (17) probably will be too complex for implementation in practical systems, the maximum capacity scheduling is interesting in that it gives the optimal combination of power allocation and scheduling, thus establishing upper performance bounds. Similar to the single-cell case treated in [1], by using the maximum capacity scheduling algorithm network throughput is optimized at the expense of fairness. However, when all users experience the same average SNR, and on average the same statistic for the interfering channel gains $G_{n, i n \neq i}$, the maximum capacity scheduling will maintain fairness over a "long enough" time horizon [16]. Should this not be the case, fairness can be restored at the price of reduced system capacity by introducing a modified scheduling policy.

Regarding an extension to systems with more than than two cells, this is ongoing work. Early results seems to indicate that the binary power control will no longer be optimal. Finally, note that a practical use of this two-cell optimization problem to larger systems, would be to cluster the cells into (carefully chosen) groups of two cells over which this optimization is carried out.

\section{NumericAl RESUlts}

In this section we present numerical results on the achievable network capacities for a two-cell wireless system utilizing link adaptation both with optimal power control and without power control. Through computer simulations both an average user pair scenario, as well as multiuser scheduling algorithms, are investigated.

\section{A. Simulation Model}

Based on the system model described in Section II, we have considered a cellular system with hexagonal layout, assuming that the users are uniformly distributed in each cell with a circumradius of $1000 \mathrm{~m}$. It is assumed that each link undergoes path loss according to the COST 231 model [17] for a small to medium-sized city, given by

$$
\begin{aligned}
P L(\mathrm{~dB}) & =46.3+33.9 \log _{10}\left(f_{\mathrm{c}}\right)-13.82 \log _{10}\left(h_{\mathrm{b}}\right) \\
& -a\left(h_{\mathrm{r}}\right)+\left(44.9-6.55 \log _{10}\left(h_{\mathrm{b}}\right)\right) \log _{10}(d),
\end{aligned}
$$

where $f_{\mathrm{c}}$ is the carrier frequency in $\mathrm{MHz}, d$ is distance between the base station and mobile user in $\mathrm{km}$, and $h_{\mathrm{b}}$ is the base station antenna height above the ground level in meters. $a\left(h_{\mathrm{r}}\right)$ is a correction factor for the mobile antenna height based on the size of the coverage area, given by

$$
a\left(h_{\mathrm{r}}\right)=\left(1.1 \log _{10}\left(f_{\mathrm{c}}\right)-0.7\right) h_{\mathrm{r}}-\left(1.56 \log _{10}\left(f_{\mathrm{c}}\right)-0.8\right),
$$

where $h_{\mathrm{r}}$ (in meters) is the mobile user antenna height above ground level. Additionally, communication is affected by
TABLE I

SIMULATION PARAMETERS

\begin{tabular}{c|c}
\hline Parameter & Value \\
\hline Cell layout & Hexagonal \\
Carrier frequency & $1800 \mathrm{MHz}$ \\
$P_{\max }$ & $1 \mathrm{~W}$ \\
Cell radius & $1000 \mathrm{~m}$ \\
BS antenna gain and height & $16 \mathrm{~dB}, 30 \mathrm{~m}$ \\
User antenna gain and height & $6 \mathrm{~dB}, 1 \mathrm{~m}$ \\
Operating temperature & $290 \mathrm{Kelvin}$ \\
Shadowing st. dev. & $10 \mathrm{~dB}$ \\
Equiv. noise BW & $1 \mathrm{MHz}$ \\
\hline
\end{tabular}

log-normal distributed shadowing and multipath fading. The log-normal shadowing is modeled as a zero-mean gaussian variable with a $10 \mathrm{~dB}$ standard deviation, and the multipath fading is considered to be Rayleigh distributed. To obtain the numerical results Monte Carlo simulations are employed with the simulation parameters listed in Table I.

\section{B. Description of Transmission Schemes}

We consider two link adaptation schemes, ideal adaptive coded modulation with and without power control, (ACMP) and (ACM) respectively. Using ACM the power at both base stations is held constant at $P_{\max }$. Based on the current received SNIR level the modulation and coding formats are selected. Allowing for power control, the ACMP scheme uses adaptive modulation to transmit at SNIR levels that are optimized according to (5). According to (5) ACMP could require that the base station is able to transmit at an infinite (continuous) number of power levels. However, since the optimal power allocation (15) has been derived to be an on/off scheme we avoid an increased complexity in transmitter design.

\section{Comparison of ACM and ACMP for An Average User Pair}

To obtain the system throughput statistics for an average user pair, we ran 10000 independent trials, in each trial drawing user locations and path gain matrix from their corresponding distributions. In Fig. 2 we have shown the system throughput $R$ from (4) as a function of $P_{1}$ and $P_{2}$ for one of these trials. It is seen that the maximum throughput is obtained by turning base station 2 off and transmitting at full power from base station 1, i.e, $\left(P_{1}, P_{2}\right)=\left(P_{\max }, 0\right)$, in agreement with (15).

Fig. 3 depicts the cumulative distribution function (CDF) of the system throughput for both ACMP and ACM. It is clear that introducing power control significantly improves the throughput performance. Specifically, ACMP and ACM have average throughputs of (both in bits/s/Hz) $\bar{R}_{\mathrm{ACMP}}=15.3$ and $\bar{R}_{\mathrm{ACM}}=12.3$, respectively.

\section{Scheduling}

We now consider multiple users per cell and study the performance of the scheduling algorithms presented in Section IV. The number of users per cell is chosen to be the same in both 


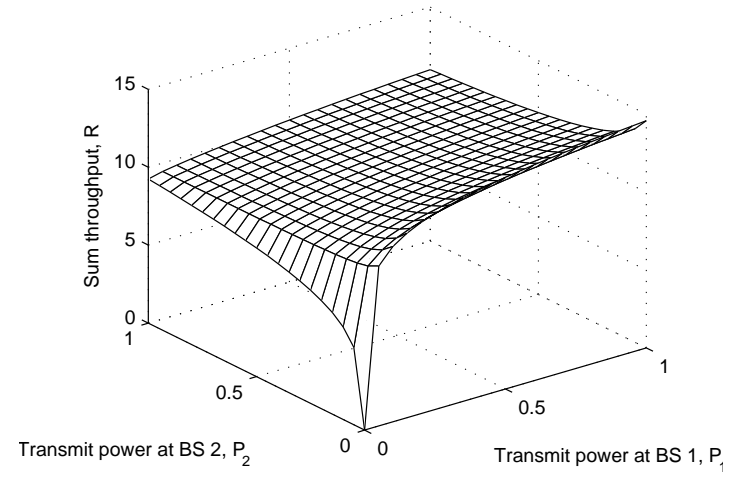

Fig. 2. Sum throughput in bits/s/Hz as a function of transmit power levels $P_{1}$ and $P_{2}$.

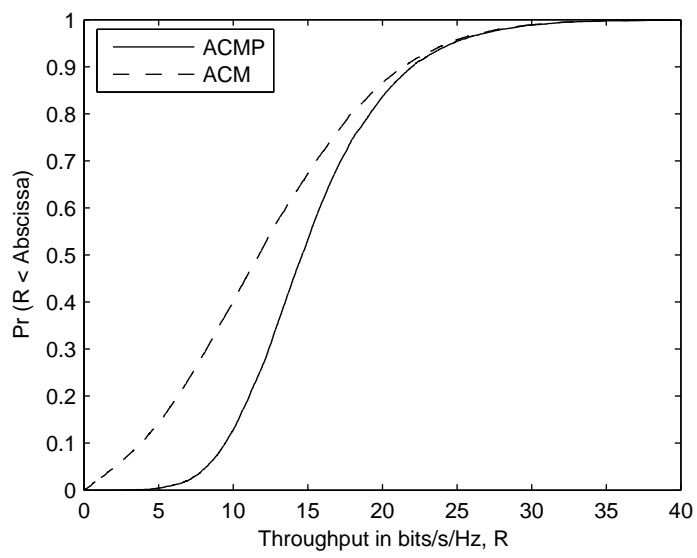

Fig. 3. CDF of the sum throughput for adaptive coded modulation with power control (solid line) and without power control (dashed line) for an average user pair.

cells, i.e., $U_{1}=U_{2}$. Based on 10000 independent trials for each number of users in the cell, Fig. 4 depicts the system capacity, for the various scheduling algorithms, measured in $\mathrm{bits} / \mathrm{s} / \mathrm{Hz}$ as a function of the number of users per cell.

This figure shows that Round Robin scheduling with power control (RR-P) clearly outperforms Round Robin scheduling without power control (RR). The system capacity for both RR schemes is independent of the number of users per cell, as expected. In fact, these scheduling algorithms are equivalent to the average user pair case treated in Section V-C, and the maximum capacity scheduling algorithm with one user per cell.

Furthermore, for two-cell maximum capacity scheduling both with power control (MAX-CAP-P) and without power control (MAX-CAP), the system capacity is an increasing function of the number of users per cell. The marked increase of capacity is due to the effects of multiuser diversity [1]. Similar observations also hold for power controlled maximum SNR scheduling (MAX-SNR-P), as well as without power control (MAX-SNR). The MAX-CAP schemes show significantly higher spectral efficiencies compared to the MAX-SNR schemes, which is because the MAX-CAP schemes search

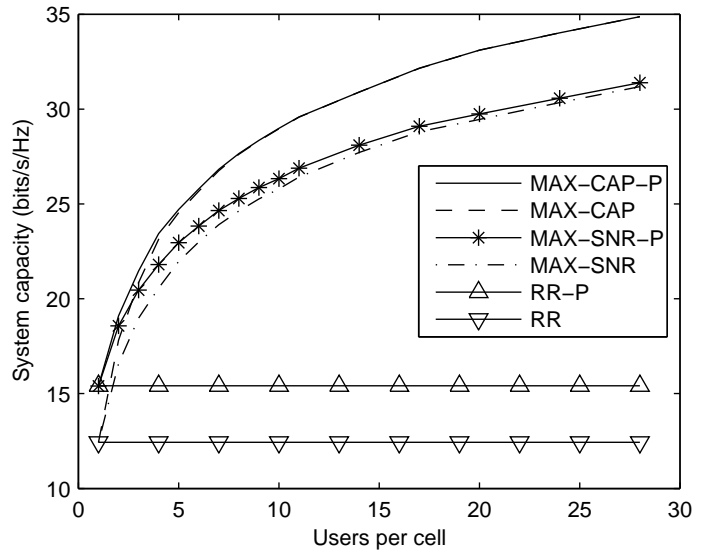

Fig. 4. System capacity in bits/s/Hz for both Round Robin and maximum capacity scheduling, as a function of the number of users per cell.

both cells simultaneously to find the best pair of users.

For a small number of users per cell the MAX-CAP-P scheme outperforms MAX-CAP. However, for an increasing number of users, these schemes have almost identical performance. This result is expected and can be explained as follows. From (4), ideally we want to schedule two users at full power that have good communication links and are not too affected by interference. Then, with an increasing number of users, the probability of finding two such users increases rapidly. This can be seen from Fig. 5 where the frequency of solutions to (5), when employing MAX-CAP-P scheduling, is plotted as a function of number of users per cell. We see that for 1 user per cell all three solutions are equiprobable in this particular simulation setting. As each cell is populated by more users the maximum capacity scheduling algorithm finds two users which receive communication emanating from base stations 1 and 2 with power $P_{\max }$, with probability close to one.

Finally, Fig. 6 shows the average power consumption for the MAX-SNR(-P) and MAX-CAP(-P) scheduling schemes, plotted as a function of the number of users. It is seen that, in addition to increasing system capacity, power control also reduces the average power consumed for both schemes, especially when the number of users in a cell is low. For an average user pair, corresponding to 1 user per cell in Fig. 6, the average power consumption is lowered by $33 \%$.

\section{CONCLUSIONS AND Discussion}

We have analyzed transmit power allocation for a two-cell wireless system, under a sum-capacity maximization criterion and peak power constraints at each base station. Given the channel gain information the proposed power scheme maximizes the network capacity. The optimal power allocation for two-cell capacity maximization is found to be surprisingly simple, transmit at full power at base station 1 , or base station 2 , or both.

An ideal link adaptation scheme with the proposed power control is compared to a similar transmission scheme without power control, for an average user pair. Our results show 


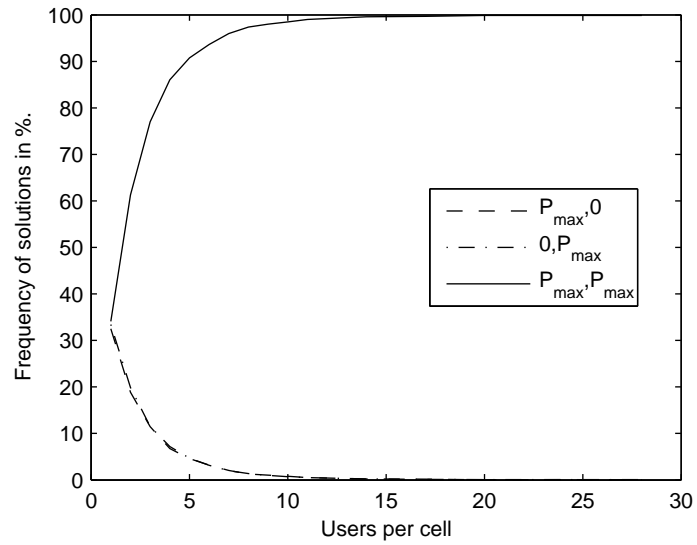

Fig. 5. Frequency of solutions to (5) versus the number of users in each cell, for two-cell maximum capacity scheduling.

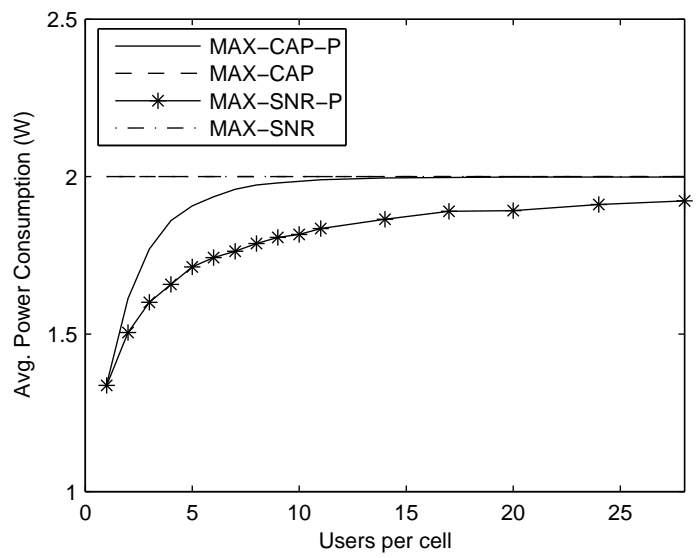

Fig. 6. Average power consumption for maximum capacity scheduling with and without power adaptation, as a function of the number of users in each cell.

that introducing power adaptation significantly increases the total throughput compared to constant power transmission. Furthermore, we study the problem of joint power control and scheduling of users from a network perspective. For Round Robin scheduling, power control substantially increases the network capacity, and at the same time reduces the average power consumption. Introducing the two-cell maximum capacity scheduling algorithm, extending the maximum SNR scheduler [1] to the two-cell case, we show that the system capacity is an increasing function of the number of users per cell. Exploiting multiuser diversity, going from 1 to 12 users per cell doubles the capacity for link adaptation with power control. For systems of more than two cells, we can apply the results presented in this paper by clustering groups of two cells, over which optmization would be effected.

In this paper we have assumed that an infinite number of rates is available. In practice however, this will not be the case. A topic for further research is to optimally design discrete rate cellular systems, following the ideas of [5], [6]. We are also currently working on extending the results to more than two cells, as well as taking into account quality of service constraints for individual users.

\section{ACKNOWLEDGEMENT}

This work was carried out when Anders Gjendemsjø was at INRIA, Sophia Antipolis, France, with financial support from NTNU, TOTAL E\&P, and the Network of Excellence NEWCOM.

\section{REFERENCES}

[1] R. Knopp and P. Humblet, "Information capacity and power control in single-cell multiuser communications," in Proc. IEEE International Conference on Communications, Seattle, WA, June 1995, pp. 331-335.

[2] J. Zander, "Performance of optimum transmitter power control in cellular radio systems," IEEE Transactions on Vehicular Technology, vol. 41, no. 1, pp. 57-62, Feb. 1992.

[3] G. J. Foschini and Z. Miljanic, "A simple distributed autonomous power control algorithm and its convergence," IEEE Transactions on Vehicular Technology, vol. 42, no. 4, pp. 641-646, Nov. 1993.

[4] Y.-H. Lin and R. L. Cruz, "Power control and scheduling for interfering links," in Proc. IEEE Information Theory Workshop, San Antonio, TX, Oct. 2004, pp. 288-291.

[5] A. Gjendemsjø, G. E. Øien, and H. Holm, "Optimal power control for discrete-rate link adaptation schemes with capacity-approaching coding," in Proc. IEEE Global Telecommunications Conference, St. Louis, MO, Nov.-Dec. 2005, pp. 3498-3502.

[6] A. Gjendemsjø, G. E. Øien, and P. Orten, "Optimal discrete-level power control for adaptive coded modulation schemes with capacityapproaching component codes," to appear in Proc. IEEE International Conference on Communications, Istanbul, Turkey, 2006.

[7] X. Qiu and K. Chawla, "On the performance of adaptive modulation in cellular systems," IEEE Transactions on Communications, vol. 47, no. 6, pp. 884-895, June 1999.

[8] A. Babaei and B. Abolhassani, "A new iterative method for joint power and modulation adaptation in cellular systems," in Proc. IFIP International Conference on Wireless and Optical Communications Networks, Dubai, United Arab Emirates, Mar. 2005, pp. 94-97.

[9] K. L. Baum, T. A. Kostas, P. J. Sartori, and B. K. Classon, "Performance characteristics of cellular systems with different link adaptation strategies," IEEE Transactions on Vehicular Technology, vol. 52, no. 6, pp. 1497-1507, Nov. 2003.

[10] S. Das, H. Wiswanathan, and G. Rittenhouse, "Dynamic load balancing through coordinated scheduling in packet data systems," in Proc. IEEE Conference on Computer Communications, San Francisco, CA, Mar.Apr. 2003, pp. 786-796.

[11] T. Park, J. Jang, O.-S. Shin, and K. B. Lee, "Transmit power allocation for a downlink two-user interference channel," IEEE Communication Letters, vol. 9, no. 1, pp. 13-15, Jan. 2005.

[12] A. Bedekar, S. Borst, K. Ramanan, P. Whitting, and E. Yeh, "Downlink scheduling in CDMA data networks," in Proc. IEEE Global Telecommunications Conference, Rio de Janeiro, Brazil, Dec. 1999, pp. 2653-2657.

[13] J. Zander and M. Frodigh, "Comment on "Performance of optimum transmitter power control in cellular radio systems"," IEEE Transactions on Vehicular Technology, vol. 43, no. 3, p. 636, Aug. 1994.

[14] J. L. Troutman, Variational calculus and optimal control: Optimization with elementary convexity, 2nd ed. New York: Springer-Verlag, 1996.

[15] D. Arora and P. Agathoklis, "Multiuser scheduling for downlink in multiantenna wireless systems," in Proc. IEEE International Symposium on Circuits and Systems, Kobe, Japan, May 2005, pp. 1718-1721.

[16] D. Gesbert and M.-S. Alouini, "Selective multi-user diversity," in International Symposium on Signal Processing and Information Technology, Darmstadt, Germany, Dec. 2003, pp. 162-165.

[17] COST Action 231, "Digital mobile radio towards future generation systems, final report," European Communities, EUR 18957, Tech. Rep., 1999. 